\title{
Coupled Motion Simulation of the Mobile Harbor and Anti-Rolling Devices in Waves
}

\author{
+Hyeon-Kyu Yoon ·Joo-Nyun Kang* ·Jae-Moon Lew** ·Seok-Joon Moon*** · Tae-Young Chung**** \\ † Dept. of Naval Archtecture \& Marine Engineering, Changwon National University, Changwon 641-773, Republic. of Korea \\ * Dept. of Naval Archtecture \& Marine Engineering, University of Strathclyde, Glasgow, G40LZ, United Kingdom \\ ** Dept. of Naval Architecture \& Ocean Engineering, Chungnam National University, Daejeon, 305-764, Republic. of Korea \\ ***, **** Korea Institute of Machinery \& Materials, Daejeon, 305-343, Republic. of Korea
}

\begin{abstract}
The Mobile Harbor $(M H)$ is a new transportation platform that can load and unload containers to and from very large container ships in the sea. This loading and unloading by crane can be performed with only very small movements of the MH in waves because $M H$ is operated outside of the harbor. For this reason, an anti-rolling tank(ART) and an active mass driving system(AMD) were designed to reduce MH's roll motion, especially at the natural frequency of MH. In the conceptual design stage, it is difficult to confirm the design result of theses anti-rolling devices without modeling and simulation tools. Therefore, the coupled MH and anti-rolling devices' dynamic equations in waves were derived and a simulation program that can analyze the roll reduction performance in various conditions, such as sea state, wave direction, and so on, was developed. The coupled equations are constructed as an eight degrees of freedom (DOF) motion that consists of MH'S six DOF dynamics and the ART'S and AMD's control variables. In order to conveniently include the ART'S and AMD's control dynamics in the time domain, MH's radiated wave force was described by an impulse response function derived by the damping coefficient obtained in the frequency domain, and wave exciting forces such as Froude-Krylov force and diffraction force and nonlinear buoyancy were calculated at every simulation time interval. Finally, the roll reduction performances of the designed anti-rolling devices were successfully assessed in the various loading and wave conditions by using a developed simulation program.
\end{abstract}

Key words : Mobile harbor, Simulation, Anti-rolling tank, Active mass driving system, Coupled equations of motion, Wave force

\section{Introduction}

The Mobile Harbor(MH) is a new transportation platform which can load and unload containers to and from very large container ships in the sea. It was originally designed to be used in order to improve oversea transportation efficiency in small conventional harbors in which a large ship cannot be at anchor. $\mathrm{MH}$ is designed as a catamaran-shaped ship, which is equipped with a precisely controlled gantry crane on the deck and the ship can transport 250 TEUs at a time(MTI et al., 2009).

Even though $\mathrm{MH}$ is operated outside of the harbor, loading and unloading by the crane can only be performed when $\mathrm{MH}$ is affected by no more than very small waves. For this reason, two types of anti-rolling devices were designed to reduce MH's roll motion, especially in resonant frequency between MH's natural roll and the modal frequency of irregular waves of sea state 3. These two types of anti-rolling devices consist of a passive anti-rolling tank (ART) and an active mass driving system(AMD)(Lee et al, 2004; Lloyd, 1989; Sellars et al, 1992). The ART reduces most of the roll motion due to waves; in addition, AMD is used in order to stabilize $\mathrm{MH}$ precisely and to allow the crane on MH to work normally. The goal of the roll reduction performance of anti-rolling devices is to achieve a $70 \%$ reduction of roll in the resonant regular beam wave condition compared to the case of a nonoperational device.

In the conceptual design stage, it is difficult to confirm the design result of the above anti-rolling devices without modeling and simulation tools. Therefore the coupled $\mathrm{MH}$ and anti-rolling devices' dynamic equations in waves were derived and a simulation program which can analyze the roll reduction performance in various conditions like sea state, wave direction, and so on, was developed. The coupled equations were constructed as an eight degrees of freedom (DOF) motion which consists of MH's six DOF dynamics, the fluid level of the right wing tank of ART, and the moving weight displacement of AMD. Simulation of ship

\footnotetext{
† Corresponding author, hkyoon@changwon.ac.kr 055)213-3683

* joonyun.Kang@gmail.com +44(141)330-2648

** jmlew@cnu.ac.kr 042)821-6628

*** sjmoon@kimm.re.kr, tychung@kimm.re.kr 042)868-7428

**** tychung@kimm.re.kr 042)868-7420
} 
motion can be performed in the frequency domain and in the time domain. It is more convenient to describe ship's motion in the time domain when additional nonlinear force components such as nonlinear buoyancy, ART's and AMD's control forces, and cargo acting force are considered in the equations of motion. In the time domain, radiated force by $\mathrm{MH}$ is described by an impulse response function derived by damping coefficient obtained in the frequency domain(Price et al, 1974; Newman, 1977). The wave exciting force, which consists of Froude-Krylov force and diffraction force, and nonlinear buoyancy were calculated at every simulation time interval.

Using the developed coupled equations of motion, the roll reduction performance of the designed ART and AMD, depending on the loading conditions of MH, were assessed based on the concept of resonant roll reduction. In addition, the roll reduction performances of the anti-rolling devices in irregular waves and against loading cargo weight were assessed.

As a result, the developed simulation tools could be used in confirming the performance of the designed anti-rolling devices if those fit into the desired goal.

\section{Equations of Motion}

\subsection{Coordinate systems}

In order to describe the motion of $\mathrm{MH}$, two kinds of coordinate systems are defined, as shown in Fig. 1. The linear displacement is represented in the earth-fixed frame denoted by $\mathrm{O}-\mathrm{XYZ}$, while the equations of motion are described in the body-fixed frame denoted by $\mathrm{O}^{-} \mathrm{xyz}$. The hydrodynamic force and moment acting on $\mathrm{MH}$ can be more easily described in the body-fixed frame than the earth-fixed frame. The origin of the body-fixed frame is the intersection of the longitudinal center line, the water line, and the midship section, and the direction of the positive $z$ axis is upwards.

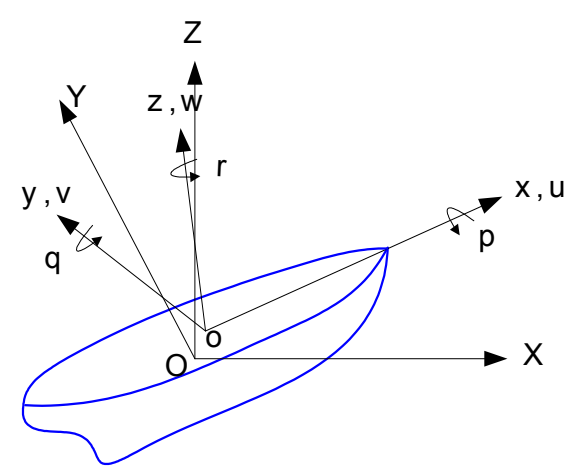

Fig. 1 Coordinate systems
In addition to the above two frames, two body-fixed frames describing the fluid level of right hand side wing tank of ART and the displacement of moving weight of AMD were adopted.

\subsection{Mobile harbor}

MH's six DOF equations of motion can be described with respect to the body-fixed frame as follows:

$$
\begin{aligned}
& \dot{m} \underline{\underline{v}}=f_{I}+f_{H}+f_{S}+f_{E} \\
& \dot{\underline{\omega}}=\underline{m}_{I}+\underline{m}_{H}+\underline{m}_{S}+\underline{m}_{E}
\end{aligned},
$$

where $m, I, \underline{v}$, and $\underline{\omega}$ are the mass, mass moment of inertia, linear velocity vector $\left([u v w]^{T}\right)$, and angular velocity vector $\left([p q r]^{T}\right)$, respectively. Subscripts $I, H, S$, and $E$ denote Coriolis and centrifugal force, hydrodynamic and hydrostatic force including wave exciting force and buoyancy, gravitational force, and external force, respectively, with which anti-rolling devices act on $\mathrm{MH} . \underline{v}$ and $\underline{\dot{\omega}}$ are the time rate of the components of $\underline{v}$ and acceleration of $\underline{\omega}$, respectively.

Coriolis and centrifugal force and moment can be described as follows:

$$
\begin{aligned}
& f_{I}=-m\left[\underline{\omega} \times \underline{v}+\dot{\omega} \times \underline{r}_{G}+\underline{\omega} \times\left(\underline{\omega} \times \underline{r}_{G}\right)\right], \\
& \underline{m}_{I}=-\left[\underline{\omega} \times(\underline{\omega})+m \underline{r}_{G} \times(\underline{\hat{v}}+\underline{\omega} \times \underline{v})\right]
\end{aligned}
$$

where $\underline{r}_{G}$ is the position vector of the center of gravity with respect to the body-fixed frame.

Since the main concern of this motion simulation is when $\mathrm{MH}$ loads or unloads cargo at sea without running, it was assumed that the hydrodynamic reaction force and moment due to ship maneuvering could be neglected. Therefore, the only hydrodynamic force is wave related terms, as described in the following:

$$
f_{H}=f_{W T}+f_{W D}+f_{W R},
$$

where subscripts $W I, W D$, and $W R$ are Froude-Krylov including hydrostatic force, diffraction, and radiation force, respectively. Froude-Krylov force can be easily calculated by integrating local pressure on MH's surface under waterline which can be obtained by incident wave potential. Wave diffraction force was calculated by superposition of sinusoidal diffraction terms with respect to every regular wave frequency which consists of irregular waves.

Radiation force which represents the terms due to MH's motion can be described by convolution integral to the 
impulse response function multiplied by the motion variable's present and past time history as follows:

$$
f_{W R}=\int_{\infty}^{\infty} h(\tau) \underline{v}(t-\tau) d \tau
$$

The impulse response function matrix $(h(t))$ can be obtained by inverse Fourier transforming of damping coefficients calculated in the frequency-domain.

Gravitational force can be easily described using the Euler transformation matrix as follows:

$$
\begin{aligned}
& f_{S}=C_{n}^{b} \underline{W} \\
& \underline{m}_{S}=\underline{r}_{G} \times\left(C_{n}^{b} \underline{W}\right)
\end{aligned},
$$

where $C_{n}^{b}$ and $\underline{W}$ are the yaw-pitch-roll Euler transformation matrix from an earth-fixed frame to a body-fixed frame and the gravity vector of $\mathrm{MH}$ described with respect to earth-fixed frame, respectively.

Finally, the forces acting on $\mathrm{MH}$ due to anti-rolling devices are described as follows;

$$
f_{E}=f_{\mu}+f_{C}+f_{R}
$$

where subscripts $\mu, C$, and $R$ are, respectively, the friction, the control due to the actuator of $\mathrm{AMD}$, and the inertia and gravitational terms of the moving water of ART and the weight of AMD. Friction and control terms will not be in the final coupled motion of equations because those are internal forces. However, those factors influence the motion of the controls of anti-rolling devices.

\subsection{Anti-rolling tank}

If the free surface effect can be neglected compared to the inertial and gravitational force of the fluid, the total fluid can be assumed to be a point mass whose center of gravity is moved following MH's motion. The shape of the real tank was simplified to have two wing tanks and one duct. The fluids in the wing tank and duct only flow vertically and

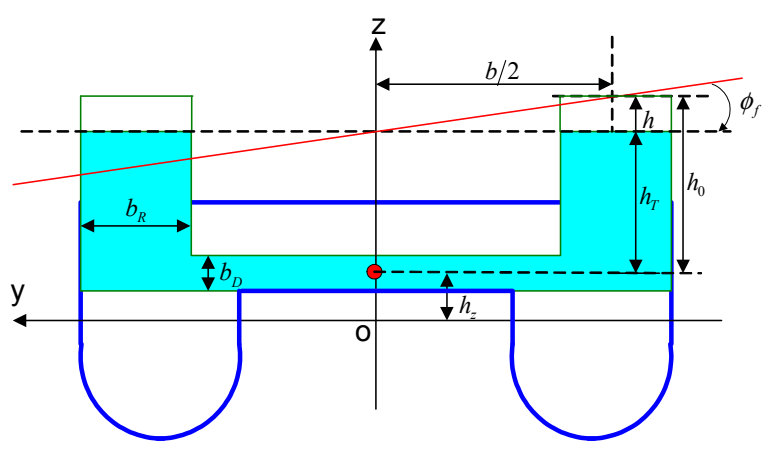

Fig. 2 ART and definitions of various symbols horizontally, respectively, without local turbulence. Therefore, the center of gravity of the fluid in the tank moves only horizontally even though the fluid in the wing tanks flows vertically.

Fig. 2 shows the ART equipped in $\mathrm{MH}$ and definitions of various symbols.

The fluid in ART is moved due to its inertia and gravity, as well as MH's motion. Therefore, the equation of the motion of the center of gravity of the fluid is described as follows:

$$
\begin{aligned}
& m_{f}\left[\underline{\underline{v}}+\underline{\omega} \times \underline{v}+\underline{r}_{f}+2 \underline{\omega} \times \underline{r}_{f}+\underline{\dot{\omega}} \times \underline{r}_{f}+\underline{\omega} \times\left(\underline{\omega} \times \underline{r}_{f}\right)\right], \\
& =f_{S_{f}}+f_{\mu_{f}}+f_{R_{f}}
\end{aligned}
$$

where $m_{f}$ and $\underline{r}_{f}$ are the mass of the fluid in ART and its position vector of the center of gravity, respectively. Subscript $f$ means the ART-related variable.

The time rate of $\underline{r}_{f}$ can be represented using the height of the fluid level of the right wing tank depicted in Fig. 2 as follows:

$$
\underline{r}_{f}=\left[\begin{array}{c}
0 \\
-\frac{\rho_{f} A_{R} b}{m_{f}} \dot{h} \\
0
\end{array}\right] .
$$

Gravitational force and moment can be described in the same manner as in Eq. (5), and friction is modeled simply as multiplying the frictional coefficient by the fluid's velocity.

As shown in Eqs. (7-8), the equation of the motion of ART can be described by $h$, and it can be represented using a non-inertial Euler equation as follows:

$$
\begin{aligned}
& \left(2 A_{R} h_{T}+\frac{A_{R}^{2}}{A_{D}} b\right) \ddot{h}-A_{R} b \dot{v}-A_{R} b\left(h_{T}-h_{z}\right) \dot{p}-A_{R} b x_{f} \dot{r}, \\
& =A_{R} b\left[\left(h_{T}+h_{z}\right) r q+\left(u r-w p+x_{f} q p\right)\right] \\
& \quad+A_{R} b g \cos \theta \sin \phi-2 A_{R} g h-\frac{\mu_{f}}{\rho_{f}} \dot{h}
\end{aligned}
$$

where $A_{R}, A_{D}$, and $x_{f}$ are cross sectional areas of the wing tank and duct and the length of ART, respectively. $g$ and $\rho_{f}$ are the gravitational acceleration and fluid density, respectively.

\subsection{Active mass driving system}

While ART is a passive type, AMD is an active type that can bring more robust improvements to the controllability. Therefore, the control force created by an actuator is added 
in the right-hand side of equations of the motion of moving weight. Fig. 3 shows $\mathrm{AMD}$ and definitions of various symbols.

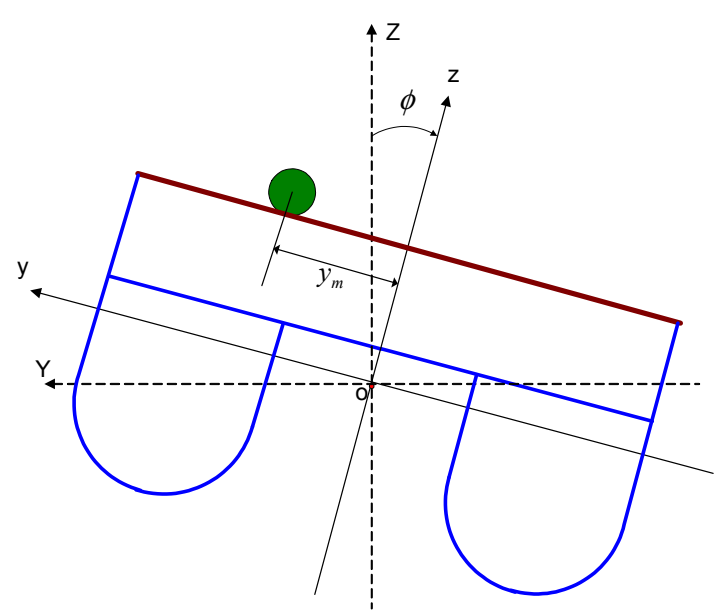

Fig. $3 \mathrm{AMD}$ and definitions of various symbols

If it is assumed that the moving weight moves horizontally only and it is a point mass, its equation of motion considering MH's motion is as follows:

$$
\begin{aligned}
& m_{m}\left[\underline{\underline{v}}+\underline{\omega} \times \underline{v}+\underline{r}_{m}^{\prime}+2 \underline{\omega} \times \underline{r}_{m}^{\prime}+\underline{\omega} \times \underline{r_{m}}+\underline{\omega} \times\left(\underline{\omega} \times \underline{r_{m}}\right)\right], \\
& =f_{S_{m}}+\underline{f}_{\mu_{m}}+\underline{f}_{C_{m}}+f_{R_{m}}
\end{aligned},
$$

where $m_{m}$ and $\underline{r}_{m}$ are the mass of the moving weight and its position vector of the center of gravity, respectively. Subscript $m$ refers to AMD-related variables. The only difference between Eq. (7) and Eq. (10) is the inclusion or exclusion of control force in the forcing term.

According to the above assumption, the time rate of $\underline{r}_{m}$ can be represented using the lateral distance of the moving weight $\left(y_{m}\right)$ as follows:

$$
\underline{r}_{m}^{\prime}=\left[\begin{array}{c}
0 \\
y_{m} \\
0
\end{array}\right]
$$

Gravitational force and moment and friction can be modeled in the same manner as in the case of ART. Control force can be considered using the following PD guidance and control algorithm:

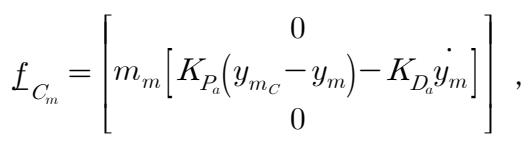

where $y_{m_{C}}=K_{P} \phi+K_{D} p$.

In Eq. (12), $K_{P_{a}}$ and $K_{D_{a}}$ are the control gains of the actuator.

The motion of the moving weight is described as its lateral distance as follows:

$$
\begin{aligned}
& m_{m}\left(\dot{v}+x_{m} \dot{r}-z_{m} \dot{p}+\ddot{y_{m}}\right) \\
& =-m_{m}\left[u r-w p+x_{m} p q-y_{m}\left(r^{2}+p^{2}\right)+z_{m} q r\right] \\
& \quad+\left(\dot{f}_{S_{m}}+f_{\mu_{m}}+f_{C_{m}}\right)_{2}
\end{aligned}
$$

where $x_{m}$ is $x$ position of $\mathrm{AMD}$ and subscript 2 is the second component of a vector.

\subsection{Cargo}

In this paper, cargo was considered only as additional weight on a specific position, so cargo can be modeled in a similar manner to the case of consideration of gravitational force and moment as follows:

$$
\begin{aligned}
& f_{c}=C_{n}^{b} \underline{W}_{c} \\
& \underline{m}_{c}=\underline{r}_{c} \times \underline{f}_{c}
\end{aligned}
$$

where $\underline{r}_{c}$ is the position vector of cargo described with respect to a body-fixed frame.

\subsection{Resultant coupled equations of motion}

According to Newton's third law, the sum of action and reaction force and moment is zero. This law can be described in this paper as follows(Yoon et al., 2004):

$$
\begin{aligned}
& f_{\mu}+\underline{f}_{\mu_{f}}+\underline{f}_{\mu_{m}}=\underline{0} . \\
& f_{C}+\underline{f}_{C_{m}}=\underline{0} \\
& f_{R}+\underline{f}_{R_{f}}+\underline{f}_{R_{m}}=\underline{0}
\end{aligned}
$$

If Eqs. (1),(7), and (10) are added to Eq. (15) and Eq. (14) is added to the resultant coupled equations of motion, the final equation can be described as follows:

$$
\begin{aligned}
& \left(m+m_{m}+m_{f}\right) \underline{\underline{v}}-\left(m_{\underline{r}_{G}}+m_{m} \underline{r}_{m}+m_{f} \underline{r}_{f}\right) \times \underline{\dot{\omega}}+m_{m} \underline{\underline{r}}_{m}+m_{f} \underline{\underline{r}}_{f} \\
& =-\left(m+m_{m}+m_{f}\right)(\underline{\omega} \times \underline{v})-\underline{\omega} \times\left[\underline{\omega} \times\left(m \underline{r}_{G}+m_{m} \underline{r}_{m}+m_{f} \underline{r}_{f}\right)\right] \\
& \quad-2 m_{m} \underline{\omega} \times \underline{\underline{r}}_{m}-2 m_{f} \underline{\omega}_{\underline{r}_{f}}+f_{S}+\underline{f}_{S_{m}}+\underline{f}_{S_{f}}+f_{H}+\underline{f}_{c}
\end{aligned}
$$

$$
\begin{aligned}
& \dot{\underline{I}}-m_{m} \underline{r}_{m} \times\left(\underline{r}_{m} \times \underline{\dot{\omega}}\right)-m_{f} \underline{r}_{f} \times\left(\underline{r}_{f} \times \underline{\dot{\omega}}\right) \\
& +\left(m \underline{r}_{G}+m_{m} \underline{r}_{m}+m_{f} \underline{r}_{f}\right) \times \underline{\dot{v}}+m_{m} \underline{r}_{m} \times \underline{r}_{m}+m_{f} \underline{r}_{f} \times \underline{\underline{r}}_{f} \\
& =-\underline{\omega} \times(\underline{I \omega})-\left(m \underline{r}_{G}+m_{m} \underline{r}_{m}+m_{f} \underline{r}_{f}\right) \times(\underline{\omega} \times \underline{v}) \\
& -2 m_{m} \underline{r}_{m} \times\left(\underline{\omega} \times \underline{r}_{m}^{\prime}\right)-2 m_{f} \underline{r}_{f} \times\left(\underline{\omega} \times \underline{r}_{f}\right) \\
& -m_{m} \underline{r}_{m} \times\left[\underline{\omega} \times\left(\underline{\omega} \times \underline{r}_{m}\right)\right]-m_{f} \underline{r}_{f} \times\left[\underline{\omega} \times\left(\underline{\omega} \times \underline{r}_{f}\right)\right] \\
& +\underline{m}_{S}+\underline{m}_{H}+\underline{r}_{m} \times \underline{f}_{S_{m}}+\underline{r}_{f} \times \underline{f}_{S_{f}}+\underline{r}_{c} \times f_{c}
\end{aligned}
$$




\section{Simulation of coupled motion}

\subsection{Mobile Harbor and anti-rolling devices}

MH is a catamaran-shaped ship that can transport 250 TEU containers and has a gantry crane on deck. Fig. 4 shows a hull shape under a designed waterline, and its principal dimensions are listed in Table 1. The designed values of $\mathrm{AMD}$ and ART are given in Table 2.

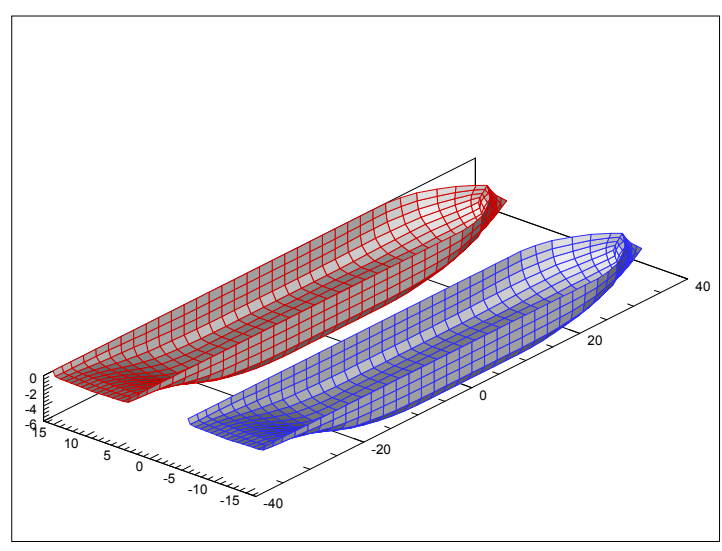

Fig. 4 Underwater hull shape of $\mathrm{MH}$

Table 1 Principal dimension of $\mathrm{MH}$

\begin{tabular}{|c|c|c|c|}
\hline \multirow{2}{*}{ Item } & \multicolumn{3}{|c|}{ Designed value } \\
\cline { 2 - 4 } & Full load & Middle load & Ballast \\
\hline LBP & $70.0 \mathrm{~m}$ & - & - \\
\hline Breadth & $33.0 \mathrm{~m}$ & - & - \\
\hline B. of side hull & $12.0 \mathrm{~m}$ & - & - \\
\hline Draft & $5.3 \mathrm{~m}$ & $4.0 \mathrm{~m}$ & $3.0 \mathrm{~m}$ \\
\hline Displacement & $7469.3 \mathrm{ton}$ & $5163.7 \mathrm{ton}$ & $3774.4 \mathrm{ton}$ \\
\hline GM & $15.6 \mathrm{~m}$ & $23.0 \mathrm{~m}$ & $37.1 \mathrm{~m}$ \\
\hline $\begin{array}{c}\text { Estimated } \\
\text { Natural frequency }\end{array}$ & $0.937 \mathrm{rad} / \mathrm{s}$ & $0.946 \mathrm{rad} / \mathrm{s}$ & $1.027 \mathrm{rad} / \mathrm{s}$ \\
\hline Speed & $8 \mathrm{knots}$ & - & - \\
\hline
\end{tabular}

Table 2 Principal dimensions of AMD and ART

\begin{tabular}{|c|c|c|}
\hline Type & Item & Value \\
\hline \multirow{4}{*}{$\begin{array}{c}\text { ART } \\
\text { Full }\end{array}$} & Breadth of wing tank $\left(b_{R}\right)$ & $2.1 \mathrm{~m}$ \\
\cline { 2 - 3 } load $)$ & Height of wing tank $\left(h_{0}\right)$ & $3 \mathrm{~m}$ \\
\cline { 2 - 3 } & Height of duct $\left(b_{D}\right)$ & $3 \mathrm{~m}$ \\
\cline { 2 - 3 } & Length of tank & $30 \mathrm{~m}$ \\
\cline { 2 - 3 } & Distance between wing tanks $(b)$ & $31.9 \mathrm{~m}$ \\
\cline { 2 - 3 } & Water height of wing tank $\left(h_{T}\right)$ & $2 \mathrm{~m}$ \\
\cline { 2 - 3 } & Height of centerline of duct $\left(h_{z}\right)$ & $4.2 \mathrm{~m}$ \\
\cline { 2 - 3 } & Damping ratio & 0.379 \\
\hline \multirow{4}{*}{ AMD } & Weight of moving weight $\left(m_{m} g\right)$ & $30 \mathrm{ton}$ \\
\cline { 2 - 3 } & Height of rail $\left(z_{m}\right)$ & $10 \mathrm{~m}$ \\
\cline { 2 - 3 } & Length of rail & $33 \mathrm{~m}$ \\
\cline { 2 - 3 } & Damping ratio & PD \\
\cline { 2 - 3 } & Control type & \\
\hline
\end{tabular}

\subsection{Added mass and damping coefficients}

Added mass and damping coefficients with respect to the frequencies of regular wave components were calculated using the three-dimensional panel method. Figs. 5-7 show the calculated added mass and damping coefficients of several loading conditions, which are described in Table 1 . In addition, Fig. 8 shows the RAO in beam sea condition.

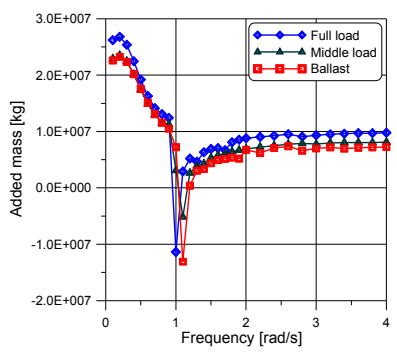

(a) Added mass

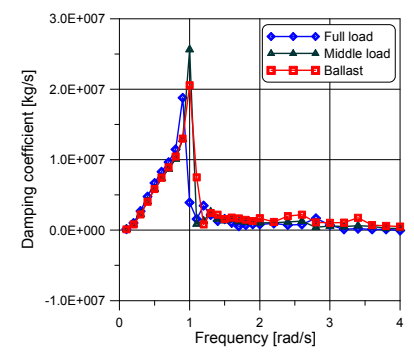

(b) Damping
Fig. 5 Heave added mass and damping coefficients

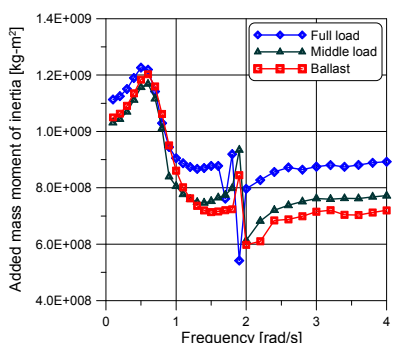

(a) Added mass

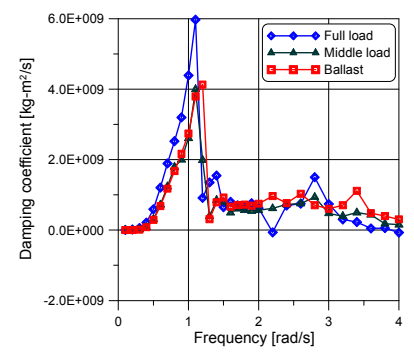

(b) Damping
Fig. 6 Roll added mass and damping coefficients

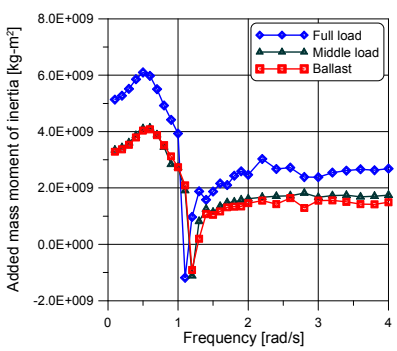

(a) Added mass

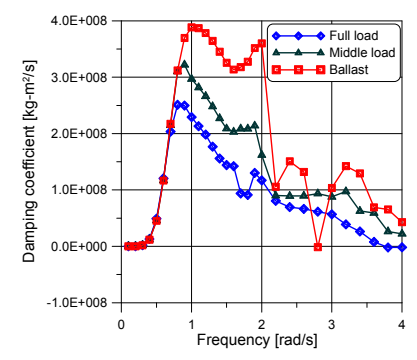

(b) Damping
Fig. 7 Pitch added mass and damping coefficients

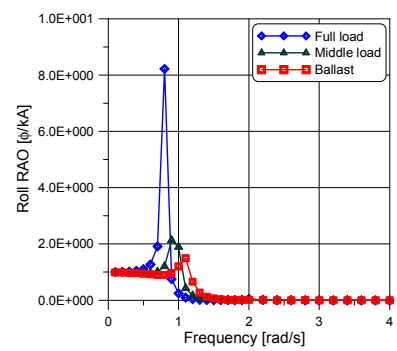

Fig. 8 Roll RAO in beam sea condition 
Compared to conventional barges, the coefficients change less smoothly because of trapped water between the side hulls. Furthermore, roll damping is very large and pitch damping is more or less small.

As shown in Fig. 8, the RAO in the full load condition reveals peak value around $0.8 \mathrm{rad} / \mathrm{s}$, which is a little different from the estimated value in Table 1. Resonant RAO of the full load is bigger than the other two loading conditions, and most of reasonable values are located lower $1.2 \mathrm{rad} / \mathrm{s}$.

\subsection{Impulse response functions}

Impulse response functions, which are used in the time domain simulations in order to describe the $\mathrm{SO}^{-}$called memory effect, can be calculated using the added mass and damping coefficients depicted in Figs. $5^{-7}$ by inverse Fourier transforms. Fig. 9 shows impulse response functions of $\mathrm{MH}$ with respect to various loading conditions. As shown in Fig. 9, past roll has less influence on present motion, while past heave and pitch continue to have influence for a long time.

\subsection{Simulation of the Mobile Harbor only}

In order to confirm the validity of the equations of the motion in the time domain, MH's motion without stabilizing was calculated in the time domain and compared to the results obtained in the frequency domain using MH's response amplitude operator (RAO)(Faltinsen, 1990).

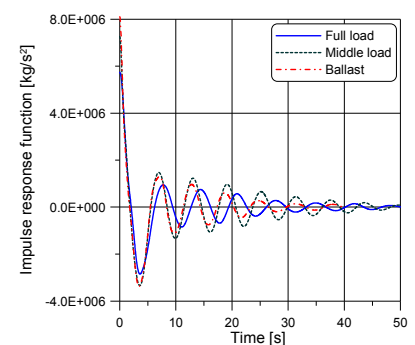

(a) Heave

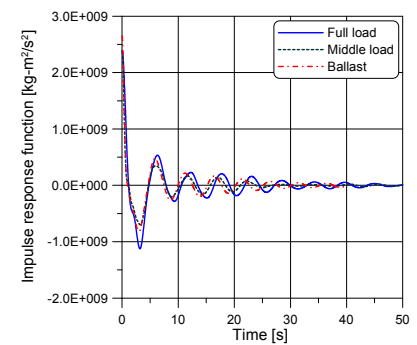

(b) Pitch

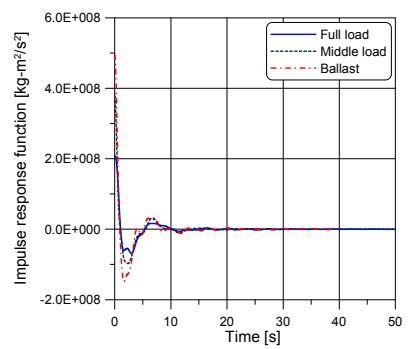

(c) Roll

Fig. 9 Impulse response functions
Fig. 10 shows the full load MH's motion responses in long crested irregular waves of sea state 3 and 4 . The regular wave frequencies and heights of both sea states are modal frequencies and significant wave heights, which are 0.84 and $0.71 \mathrm{rad} / \mathrm{s}$ in frequencies and 0.88 and $1.88 \mathrm{~m}$ in wave heights, respectively. And those values are the same after here in this paper.

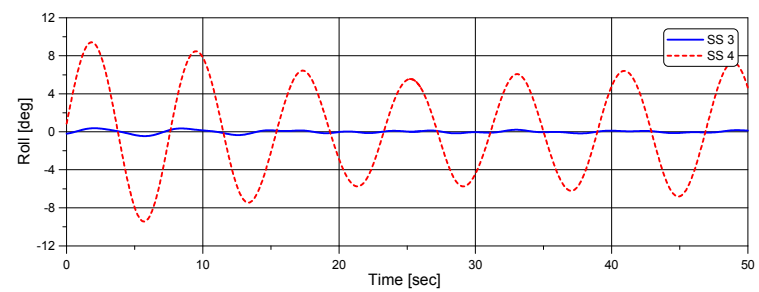

(a) Frequency domain

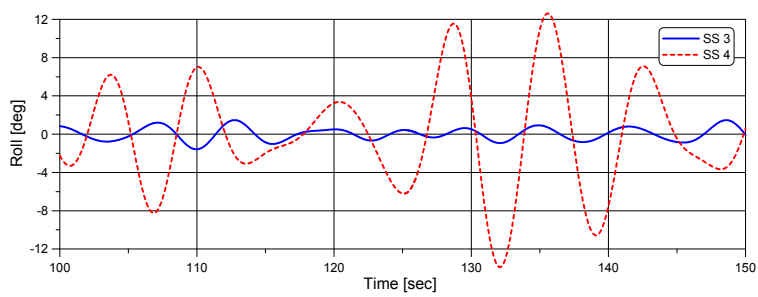

(b) Time domain

Fig. 10 Roll of the full load $\mathrm{MH}$ in irregular waves of sea state 3 and 4

As shown in Fig. 10, the maximum roll calculated in the time domain is larger than the one in the frequency domain. The main reason is that the calculation conditions are a little different. In the frequency domain, $\mathrm{MH}$ can keep its position because RAO reflects only linear oscillating terms, while in the time domain, since there are no restoring forces and moments in the surge, sway, and yaw, those modes were intentionally blocked to be motionless. Even though the conditions were different, the RMS rolls are similar in sea state 4 . In addition, the several reasons why the roll in sea state 4 is much larger than in sea state 3 are that wave amplitude in sea state 4 is over twice amplitude of sea state 3 , the sensitivity of the response of the $\mathrm{MH}$ when the $\mathrm{MH}^{\prime} \mathrm{s}$ natural frequency is similar to the wave modal frequency, and the $\mathrm{MH}$ is more responsible under low frequencies as shown in Fig. 8. The larger sea state is, the more wave energy is concentrated to the lower frequencies.

\subsection{Coupled motion simulation}

MH's motions controlled by ART and AMD were simulated using Eqs. (9), (13), (16), and (17) in regular and irregular waves. 
Figs. 11 and 12 show the time series of the roll of the full load $\mathrm{MH}$, the fluid level of the right wing tank of ART, and the distance of the moving weight of AMD in regular wave of resonant roll frequency and irregular waves of sea state 4. Anti-rolling devices control $\mathrm{MH}$ after 100 and 250 seconds in regular wave and irregular waves, respectively. The resonant roll frequency means the natural frequency of $\mathrm{MH}$ at which the RAO is peak as shown in Fig. 8, which is around $0.8 \mathrm{rad} / \mathrm{s}$. In this case that the frequency of regular wave is the same as the natural frequency of $\mathrm{MH}$, roll of $\mathrm{MH}$ is the most severe among any other cases.

As shown in Figs. 11 and 12, anti-rolling devices work properly following MH's roll, and the coupled equation of motion can simulate those motions. While the ART and AMD work well in the regular wave condition as shown in Fig. 11, those performances are reduced in the irregular wave condition. The main reason is that the time rate of the wave elevation in the irregular wave condition is randomly changed compared to the ones in the regular wave condition. Therefore, the roll reduction performance in the irregular condition is lower than the one in the regular wave

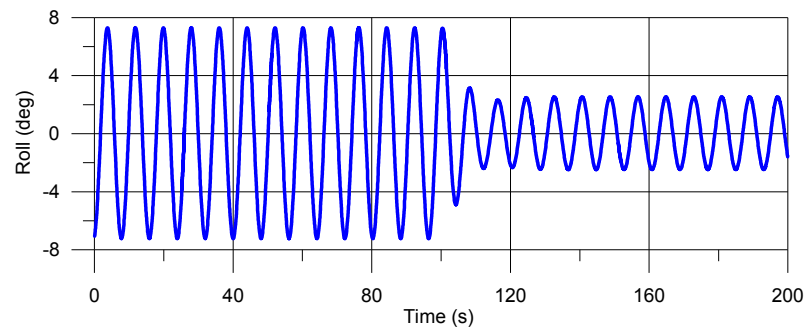

(a) Roll

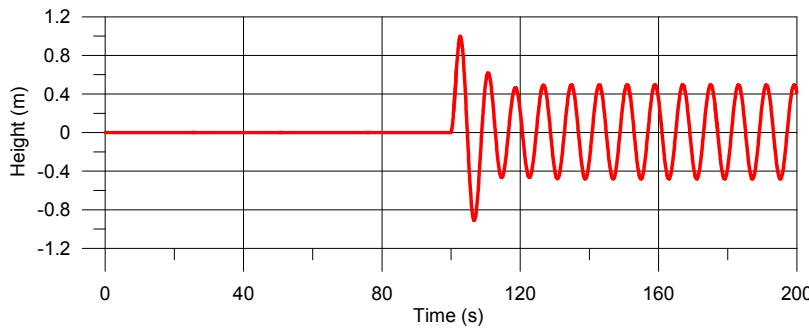

(b) Fluid level of wing tank of ART

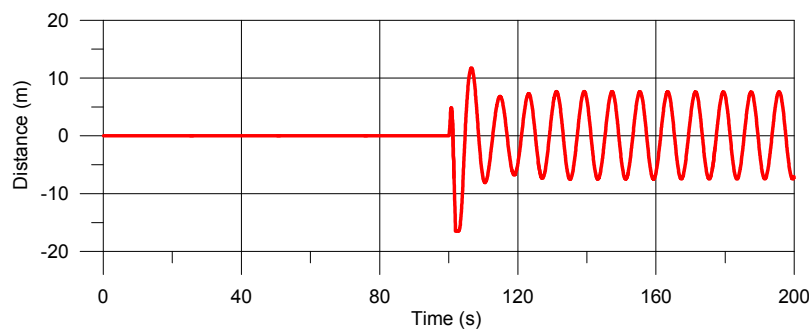

(c) Distance of moving weight of AMD

Fig. 11 Time series of the full load $\mathrm{MH}$ and control variables in regular wave when motion is controlled condition and the magnitudes of controls which are calculated by PD control laws of the ART and AMD are larger in case of the irregular wave condition. The roll reduction performance of anti-rolling devices will be assessed in detail later.

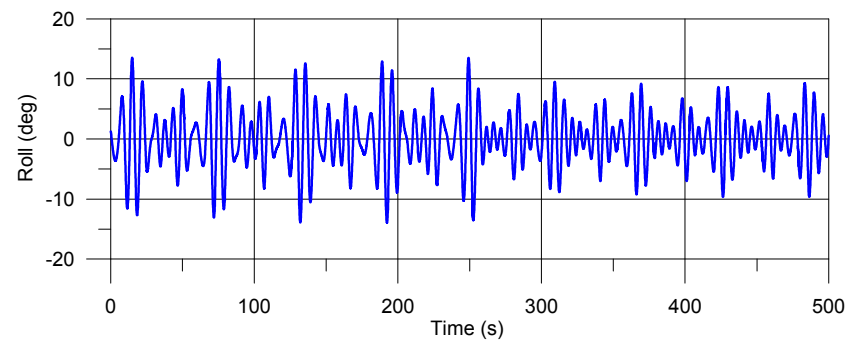

(a) Roll

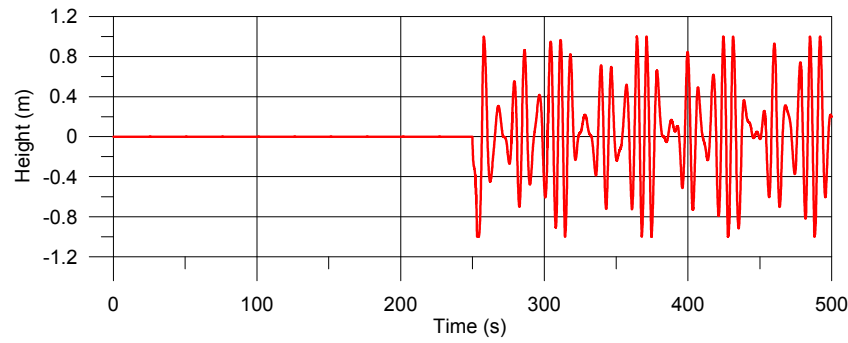

(b) Fluid level of wing tank of ART

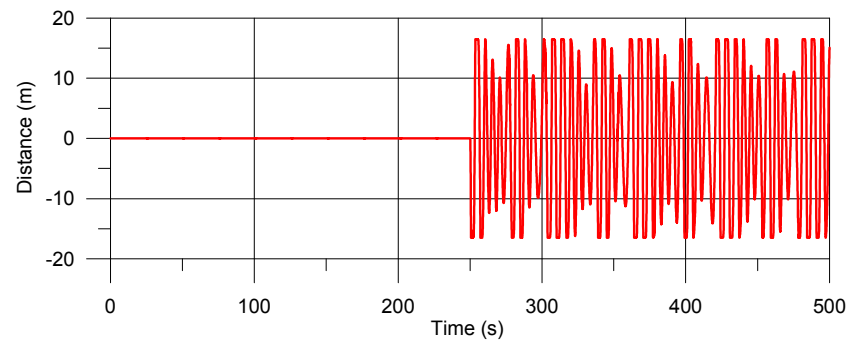

(c) Distance of moving weight of AMD

Fig. 12 Time series of the full load $\mathrm{MH}$ and control variables in irregular waves when motion is controlled

\subsection{Influence of cargo loading}

In order to confirm the cargo model described in Eq. (14), a scenario in which the gantry crane lifts an forty-foot- equivalent (FEU) container on a large container ship and keeps the state was assumed. Fig. 13 shows the time series of the roll of $\mathrm{MH}$ and control variables of ART and AMD.

Since ART is a passive device, the initial roll increases compared to the roll without control. However, $\mathrm{MH}$ stabilized quickly after just one rolling period. AMD can reduce the first roll amplitude, and if the gain is tuned more accurately, it is expected that its capability would be increased. 


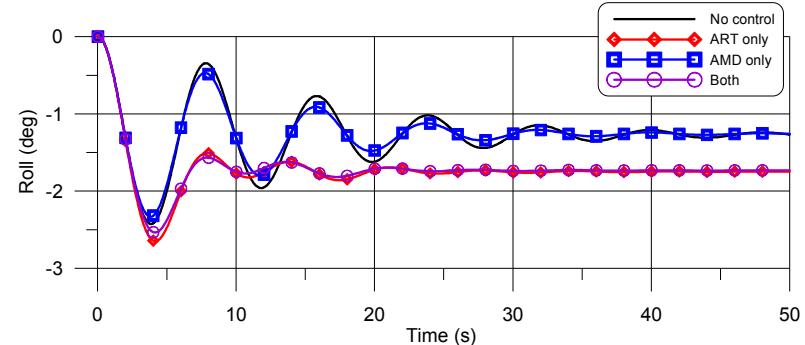

(a) Roll

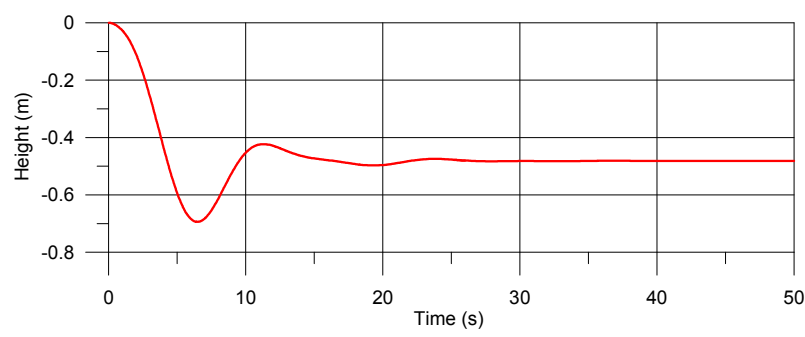

(b) Fluid level of wing tank of ART

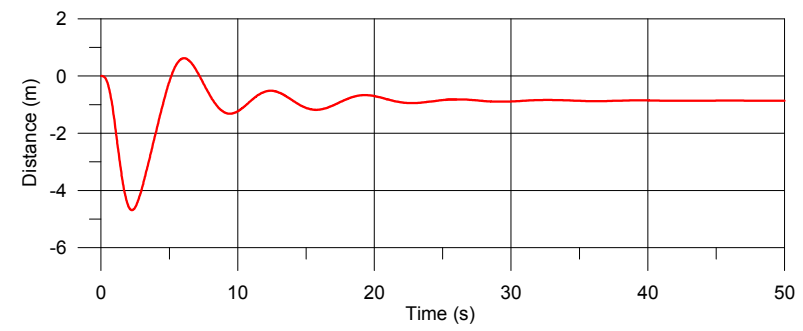

(c) Distance of moving weight of AMD

Fig. 13 Time series of the full load $\mathrm{MH}$ and control variables when 48 ton cargo is loaded

\section{Roll reduction performance}

\subsection{Resonant roll reduction performance}

Resonant roll reduction performances were assessed depending on the loading conditions. Resonant frequency is changed if the loading condition is changed. Therefore, at first, resonant frequency was found, and then the motions of $\mathrm{MH}$ were simulated following the control options such as ART only, AMD only, and both activated.

Tables 3 and 4 summarize the resonant roll reduction performances in the cases of the full load and the middle

Table 3 Resonant roll reduction performance in the case of the full load $\mathrm{MH}$

\begin{tabular}{|c|c|c|c|}
\hline \multirow{2}{*}{ Control option } & \multicolumn{2}{|c|}{ Normalized roll $(\phi / k A)$} & \multirow{2}{*}{$\begin{array}{c}\text { Reduction } \\
\text { (\%) }\end{array}$} \\
\cline { 2 - 3 } & w/o control & w/ control & \\
\hline ART only & 4.67 & 1.80 & 61.45 \\
\hline AMD only & 4.67 & 3.67 & 21.42 \\
\hline Both & 4.67 & 1.62 & 65.23 \\
\hline
\end{tabular}

load, respectively. In these tables, roll is normalized using the regular wave slope which is described by multiplying the wave number $(k)$ by the wave amplitude $(A)$.

Table 4 Resonant roll reduction performance in the case of the middle load $\mathrm{MH}$

\begin{tabular}{|c|c|c|c|}
\hline \multirow{2}{*}{ Control option } & \multicolumn{2}{|c|}{ Normalized roll $(\phi / k A)$} & Reduction \\
\cline { 2 - 3 } & w/o control & w/ control & (\%) \\
\hline ART only & 1.65 & 1.23 & 25.80 \\
\hline AMD only & 1.65 & 1.32 & 20.26 \\
\hline Both & 1.65 & 1.05 & 36.36 \\
\hline
\end{tabular}

The design of ART adopted for the middle load condition was slightly different from the one for the full load whose dimensions listed in Table 2 in order to fit ART's natural frequency into the middle load MH's natural frequency.

In terms of Table 3, designed anti-rolling devices achieve the desired performance in the full load condition. In the case of the middle load condition in which the metacenter is very high, the resulting natural frequency is also large. For this reason, it was difficult to design possible size of ART, and it influenced on mismatching of natural frequencies between $\mathrm{MH}$ and ART. This deteriorated the $\mathrm{ART}^{\prime} \mathrm{s}$ roll reduction performance.

\subsection{Roll reduction performance in irregular waves}

The roll reduction performance in irregular waves of sea state 4 were calculated and summarized in Tables 5 and 6 .

A fin stabilizer, which is the most effective stabilizer, is known to reduce $40-50 \%$ of the RMS roll in irregular waves(Lee et al, 2004). Therefore, it seems that the currently designed anti-rolling devices would be designed normally, and it is also expected that the performance will improve if the gains of $\mathrm{AMD}$ are tuned deliberately and $\mathrm{ART}$ is redesigned for wave modal frequency in the case of middle load $\mathrm{MH}$.

Table 5 Roll reduction performance in irregular waves of sea state 4 in case of full load $\mathrm{MH}$

\begin{tabular}{|c|c|c|c|}
\hline \multirow{2}{*}{ Control option } & \multicolumn{2}{|c|}{ RMS Roll $\left(^{\circ}\right)$} & \multirow{2}{*}{$\begin{array}{c}\text { Reduction } \\
\text { (\%) }\end{array}$} \\
\cline { 2 - 4 } & w/o control & w/ control & ART only \\
\hline AMD only & 5.33 & 4.23 & 20.67 \\
\hline Both & 5.33 & 5.05 & 5.24 \\
\hline
\end{tabular}


Table 6 Roll reduction performance in irregular waves of sea state 4 in case of middle load $\mathrm{MH}$

\begin{tabular}{|c|c|c|c|}
\hline \multirow{2}{*}{ Control option } & \multicolumn{2}{|c|}{ RMS Roll $\left(^{\circ}\right)$} & \multirow{2}{*}{$\begin{array}{c}\text { Reduction } \\
\text { (\%) }\end{array}$} \\
\cline { 2 - 3 } & w/o control & w/ control & \\
\hline ART only & 7.64 & 6.96 & 8.89 \\
\hline AMD only & 7.64 & 7.30 & 4.49 \\
\hline Both & 7.64 & 6.22 & 18.53 \\
\hline
\end{tabular}

\section{Conclusion}

In this paper, the coupled equations of the motion of the Mobile Harbor (MH), which is a new conceptual transportation system, and anti-rolling devices were established. In addition, cargo force was simply modeled in the derived equations in order to confirm the possibility of loading and unloading. This modeling was carried out by applying coupling equations that were established by Newton's third law on the contact points of the $\mathrm{MH}$ and anti-rolling devices after establishing equations of the motion of $\mathrm{MH}$ and anti-rolling devices, independently. The established time domain equations could be useful if new devices are added or additional nonlinear force model are included.

The resonant roll reduction performance of designed anti-rolling devices, which were the anti-rolling tank (ART) and active mass driving system (AMD), were assessed using a simulation algorithm based on developed coupled equations of motion. In addition, the roll reduction performances in irregular waves were assessed with respect to the loading conditions. It seemed that the designed anti-rolling devices were suitable for the desired performance. If the gains of AMD are tuned deliberately, the roll reduction performance will improve.

Finally, it is expected that the developed simulation algorithm can be used successfully in the conceptual design phase of anti-rolling devices in order to confirm those roll reduction performances.

\section{Acknowledgements}

This work was supported by the project "Development of anti-rolling devices of the Smart Carrier," which is sponsored by the Ministry of Education, Science and Technology of the Republic of Korea.

\section{References}

[1] Faltinsen, O.M.(1990), "Sea Loads on Ships and Offshore Structures", Cambridge University Press, pp. 37-68

[2] Lee, G.J. and Yoon, H.K(2004).. "Technical Report: Development of Technology for Attitude Control System of Ships on Waves", MOERI/KORDI, UCN00700-04072

[3] Lloyd, A.R.J.M.(1989), "SEAKEEPING: Ship Behavior in Rough Weather", ELLIS HORWOOD LIMITED, pp. 377-397

[4] MTI and 21st Century Shipbuilding Co., Ltd.(2009), "Conceptual design of MH A1-250", Technical report.

[5] Newman, J.N.(1977), "Marine Hydrodynamics", MIT Press, pp. 307-311

[6] Price, W.C. and Bishop, R.E.D.(1974), "Probabilistic theory of ship dynamics", Chapman and Hall, London, pp. $193-219$

[7] Sellars, F.H. and Martin, J.P.(1992), "Selection and Evaluation of Ship Roll Stabilization Systems", Marine Technology, Vol.29, No.2, pp. 84-101

[8] Yoon, H.K., Lee, G.J., and Lee, C.M. (2004), "Modeling and Simulation of a Ship with Anti-Rolling Devices in Waves", Journal of Korean Navigation and Port Research, Vol.28, No.5, pp. 347-352

Received 4 May 2010

Revised 25 June 2010

Accepted 26 June 2010 